age, gender, disease duration, seropositivity, $\mathrm{HAQ}$ and $\mathrm{CDAl}$ at $\mathrm{BL}$, number of previous CsDMARD and biologic DMARD (bDMARD), glucocorticosteroid and calendar year of treatment initiation. Missing data on covariates were imputed using multiple imputation with chained equations.

Results: A total of 4748 patients were eligible, including 585 who received TCZ mono and 4163 who received TNFi combo. Patients who received TCZ mono were older with a longer disease duration, more previous bDMARDs and less glucocorticosteroids at baseline (Table 1) compared with patients who received TNFi combo. The crude median retention for TCZ mono was 1.82 years $(95 \%$ Cl: 1.59-2.09) and 1.54 years (95\% Cl: $1.43-1.64)$ for TNFi combo, $(P=0.65)$. Causes of discontinuation differed between TCZ mono and TNFi combo $(P<0$. 001): TCZ mono stopped more frequently for ineffectiveness ( $25.7 \%$ vs. $13.8 \%)$ and TNFi combo stopped more frequently for safety issues (18.3\% vs. $12.8 \%)$. In a country-stratified, covariate-adjusted analysis, we found that hazards of discontinuation were significantly lower among patients who received TCZ mono (HR: 0.71, $P<0.001$ ). More previous treatment with bDMARDs and a greater HAQ and CDAl at BL were significantly associated with greater risk of discontinuation.

Table 1. Baseline characteristics.

\begin{tabular}{|c|c|c|c|}
\hline & $\begin{array}{l}\text { TCZ mono } \\
(\mathrm{N}=585)\end{array}$ & $\begin{array}{l}\text { TNFi combo } \\
(\mathrm{N}=4163)\end{array}$ & $P$ \\
\hline Age, yr, median [IQR] & $\begin{array}{l}57.8[44.2-65.5], \\
\mathrm{n}=585\end{array}$ & $\begin{array}{l}54.3[44.2-61.7], \\
\mathrm{n}=4161\end{array}$ & $<0.001$ \\
\hline Female gender, $\mathrm{N}(\%)$ & $\begin{array}{l}485(82.9 \%) \\
\mathrm{n}=585\end{array}$ & $\begin{array}{l}3333(80.1 \%) \\
\mathrm{n}=4161\end{array}$ & 0.12 \\
\hline Disease duration, yr, median [IQR] & $\begin{array}{l}9.7[4.5-16.7], \\
\mathrm{n}=569\end{array}$ & $\begin{array}{l}7.8[3.3-14.3] \\
\mathrm{n}=3575\end{array}$ & $<0.001$ \\
\hline $\begin{array}{l}\text { Seropositivity (RF and/or ACPA), } \\
\mathrm{N}(\%)\end{array}$ & $\begin{array}{l}445(83.6 \%) \\
\mathrm{n}=532\end{array}$ & $\begin{array}{l}2573(81.0 \%) \\
\mathrm{n}=3175\end{array}$ & 0.17 \\
\hline $\begin{array}{l}\text { N previous bDMARDs, } N(\%) \\
1 \\
2 \\
\geq 3 \\
\end{array}$ & $\begin{array}{l}250(42.7 \%) \\
206(35.2 \%) \\
129(22.1 \%)\end{array}$ & $\begin{array}{l}2882(69.2 \%) \\
526(12.6 \%) \\
755(18.1 \%)\end{array}$ & $<0.001$ \\
\hline Glucocorticosteroids, $\mathrm{N}(\%)$ & $\begin{array}{l}193(33.0 \%), \\
\mathrm{n}=585\end{array}$ & $\begin{array}{l}2487(59.7 \%), \\
n=4163\end{array}$ & $<0.001$ \\
\hline $\begin{array}{l}\text { Concomitant csDMARD, N (\%) } \\
\text { MTX } \\
\text { MTX + other } \\
\text { Other }\end{array}$ & $\begin{array}{l}-- \\
-- \\
--\end{array}$ & $\begin{array}{l}1766(42.4 \%) \\
1291(31.0 \%) \\
1106(26.6 \%)\end{array}$ & -- \\
\hline CDAI, mean (SD) & $\begin{array}{l}23.2(16.1) \\
\mathrm{n}=322\end{array}$ & $\begin{array}{l}21.9(14.7) \\
\mathrm{n}=3021\end{array}$ & 0.25 \\
\hline HAQ, mean (SD) & $\begin{array}{l}1.4(0.7) \\
\mathrm{n}=226\end{array}$ & $\begin{array}{l}1.1(0.7) \\
n=2429\end{array}$ & $<0.001$ \\
\hline
\end{tabular}

Conclusions: In routine care across 9 European countries, TCZ mono retention is better than TNFi combo in patients with RA who were TNFi-IR.

Acknowledgements: Funding by F. Hoffmann-La Roche/Genentech.

Disclosure of Interest: K. Lauper: None declared, D. Nordström Grant/research support from: AbbVie, BMS, MSD, Pfizer, Roche and UCB, Consultant for: AbbVie, BMS, MSD, Roche, UCB and Pfizer, K. Pavelka Grant/research support from: AbbVie, Roche, Medis, MSD and Pfizer, Consultant for: AbbVie, Roche, Amgen, MSD, BMS, UCB and Egis, V. Hernandez: None declared, M. J. Santos: None declared, Z. Rotar: None declared, F. lannone: None declared, C. Codreanu: None declared, G. Lukina Consultant for: BMS, Roche, MSD, AbbVie and Pfizer, S. Gale Employee of: Genentech, K. Sarsour Employee of: Genentech, A. Pethoe-Schramm Employee of: F. Hoffmann-La Roche, D. Courvoisier: None declared, C. Gabay Grant/research support from: AB2 Bio, AbbVie, Actelion, BMS, Debiopharm, MSD, Novartis, Pfizer, Regeneron, Roche, Sanofi and UCB, Consultant for: AB2 Bio, AbbVie, Actelion, BMS, Debiopharm, MSD, Novartis, Pfizer, Regeneron, Roche, Sanofi and UCB

DOI: 10.1136/annrheumdis-2017-eular.6503

\section{SAT0207 LONG-TERM EFFECT OF BIOLOGICAL THERAPIES ON BONE MINERAL DENSITY (BMD) FOR RA PATIENTS COMPARED TO PATIENTS TREATED BY SYNTHETIC DMARD OVER AN 8-YEAR FOLLOW-UP}

L. Bustamente $^{1}$, V. Breuil ${ }^{2}$, R. Fabre ${ }^{3}$, L. Euller-Ziegler ${ }^{2}$, C. Pradier ${ }^{3}$, C. Roux ${ }^{1}$ ${ }^{1}$ Rheumatology, University Nice, Sofia Antipolis, Nice, ${ }^{2}$ Rheumatology; ${ }^{3}$ Santé publique, University Nice, Sofia Antipolis, Nice Cedex 1, France

Background: Secondary osteoporosis is a comorbidity of Rheumatoid Arthritis (RA). Previous studies have suggested that biological therapies may reduce the rate of generalized bone loss in RA. Most of them focus on the short-term effect of Tumor Necrosis factor alpha (TNF $\alpha$ ) inhibitors while the long-term effect of biological therapies is rarely studied.

Objectives: Our primary aim was to analyze the long-term effect of biological therapies on bone mineral density (BMD) for RA patients compared to patients treated by synthetic DMARD over an 8-year follow-up.

Methods: Patients were selected from a prospective, observational cohort of RA patients meeting the ACR/EULAR 2010 settings in Nice University Hospital between 2001 and 2016. BMD was assessed before the introduction of the biological therapy and during the follow-up. Two groups were studied: patients treated by biological therapies (TNF $\alpha$ inhibitors, Tocilizumab, Abatacept, Rituximab or Anakinra) and patients treated by synthetic DMARD only. Demographic, disease and treatment data were collected at each visit and BMD of the lumbar spine, femoral neck and total hip were assessed using dual energy $\mathrm{X}$-ray absorptiometry (DXA) at baseline and after 1, 2, 3, 5 and 8 years.

Results: A total of 181 patients with active RA starting a biological therapy were included versus 131 patients treated by synthetic DMARD. In both groups, the $B M D$ of the lumbar spine, femoral neck and total hip remained stable after a 2-year follow-up (respectively $-0.37 \%, p=0.66$ versus $+0.02 \%, p=0.83,-3.70 \%, p=0.77$ versus $-5.35 \%, p=0.74$ and $-3.31 \%, p=0.45$ versus $-4.84 \%, p=0.16$ ), while a significant bone loss was found between initial measurement and 3,5 and 8 -yearfollow-up at femoral neck and total hip level. There was no significant difference between patients whether treated by biological or non-biological DMARD neither over the $0-1$-year period nor over the $0-2,0-3,0-5$ and $0-8$-periods. Bone loss in patients treated by Tocilizumab were statistically lower at femoral neck level compared with TNF $\alpha$ inhibitors $(p=0.02)$, Abatacept $(p=0.02)$ and Rituximab $(p=0.02)$, but also at total hip level, in comparison with TNF $\alpha$ inhibitors $(p=0.05)$ and Abatacept $(\mathrm{p}=0.05)$.

Conclusions: This study is the first to assess the effects of biological RA therapies as compared to synthetic DMARD ones. It highlights the protective effect of both biological and non-biological DMARD on bone loss during the first two years of treatment with no significant difference between them. Our results suggest that the effects of RA treatments depend on the inflammatory and disease activity which must be monitored clearly. Tocilizumab seems to be more effective than the other biological therapies, but further studies are necessary to confirm or infirm this tendency.

Disclosure of Interest: None declared

DOI: 10.1136/annrheumdis-2017-eular.2851

\section{SAT0208 CYCLING VERSUS SWAPPING IN PATIENTS WITH RHEUMATOID ARTHRITIS WITH AN INADEQUATE RESPONSE TO AT LEAST ONE TUMOR NECROSIS FACTOR ALPHA INHIBITOR: A SYSTEMATIC REVIEW AND META-ANALYSIS OF OBSERVATIONAL STUDIES}

M.A. Lopez-Olivo ${ }^{1}$, A. Matusevich ${ }^{2}$, M.E. Suarez-Almazor ${ }^{1} .{ }^{1}$ General Internal Medicine, The University of Texas, MD Anderson Cancer Center; ${ }^{2}$ School of Public Health, The University of Texas, Health Science Center at Houston, Houston, United States

Background: In patients with rheumatoid arthritis (RA) who do not respond or lose response, opinions are divided on whether it is better to try an alternative TNFi (cycling) or switch to a therapy with a different mode of action (swapping). Objectives: To compare the efficacy and safety of the cycling versus the swapping strategies.

Methods: We searched 4 electronic databases, sources of gray literature, and bibliographic references of relevant articles for observational studies evaluating the efficacy and safety of targeted therapies in adult RA patients who failed to respond to at least one TNFi. Studies were excluded if they were single-arm or had insufficient data to evaluate the outcomes of interest. Two independent reviewers selected studies, extracted data and evaluated study quality using the Newcastle-Ottawa Scale (NOS). Our primary outcome measure was change in Disease Activity Score of 28 joints (DAS28). We also evaluated the modified American College of Rheumatology $20 \%, 50 \%$ and $70 \%$ response criteria (mACR which excludes acute phase reactants) and total serious adverse events. All analyses were based on the random-effects model.

Results: Of 33,716 citations, 24 observational studies ( $\mathrm{n}=10,074$ patients) representing 14 countries, met the inclusion criteria. Eight were conference abstracts. Most publications (13 of 24) were based on registries. Most studies had a NOS score equal to or greater than 7 (out of 9) with comparability being the weakest domain. The mean age of patients was 48.7-62.8 years, the majority were females $(78 \%)$ with a disease duration of $6-17.3$ years and a baseline disability score $0.6-2.0$. Sixteen studies evaluated cycling versus swapping directly of which 13 were suitable for analysis. Most compared TNFi to rituximab (10 of 13) with two studies investigating tocilizumab or abatacept and one comparing non-TNFi as a group. Other comparisons reported were: (i) cycling vs. conventional disease modifying antirheumatic drugs (cDMARDs), (ii) swapping vs. cDMARDs, (iii) cycling vs. another cycling alternative, (iv) swapping vs. another swapping alternative, (v) swapping monotherapy vs. swapping combined with cDMARDs, and (vi) combination of TNFi and non-TNFi vs. TNFi alone. At 12 and 24 weeks, DAS28 score improved significantly in those swapping compared to those cycling (mean difference (MD) $0.89,95 \%$ confidence interval (CI) 0.05 to 1.74 and MD $0.3495 \% \mathrm{Cl}: 0.2,0.48$; respectively). Similar results were observed for the mACR50 favoring the swapping strategy at 24 weeks (OR $=1.4595 \% \mathrm{Cl}$ : $1.06,1.98)$. At 52 weeks no difference was observed. No statistically significant differences were observed between groups in the odds of achieving DAS28 remission, mACR20 or mACR70, or experiencing a serious adverse event.

Conclusions: Current evidence from observational studies shows greater improvements with the swapping strategy compared with the cycling strategy in terms of efficacy for RA patients failing their first TNFi. No differences were observed regarding safety. Data were not available for anakinra, certolizumab pegol, golimumab, or tofacitinib.

Acknowledgements: Funding for this project was provided by the Rheumatology Research Foundation Investigator Award.

Disclosure of Interest: None declared 
DOI: 10.1136/annrheumdis-2017-eular.6490

\section{SAT0209 OBSERVATIONAL STUDY ON THE EFFECTS OF IL-6 INHIBITOR THERAPY ON MYOSTATIN IN PATIENTS WITH RHEUMATOID ARTHRITIS}

M.J. Chapman ${ }^{1}$, R.P. Narayanan ${ }^{2}$, A. Cross $^{3}$, R. Moots ${ }^{3}$, J. Wilding $^{2}$, N. Goodson ${ }^{3} .{ }^{1}$ Department of Rheumatology, Aintree University Hospital; ${ }^{2}$ Department of Obesity and Endocrinology; ${ }^{3}$ Institute of Ageing and Chronic Disease, University of Liverpool, Liverpool, United Kingdom

Background: Rheumatoid cachexia $(\mathrm{RC})$, a phenotype of increased adiposity, reduced lean mass and insulin resistance can occur in rheumatoid arthritis (RA). It is most prevalent in those with high disease activity and is associated with increased cardiovascular morbidity and mortality. Tocilizumab (TCZ), a monoclonal anti-IL-6 antibody, successfully reduces disease activity in RA. It has also been associated with improved insulin sensitivity, increased lean mass and hyperlipidaemia. The serum concentration of myostatin, a myokine released by skeletal muscle, is increased in most disease states associated with cachexia. Moreover, its knockout and inhibition increase lean mass. However, its role in RA has not been fully elucidated.

Objectives: To investigate the effect of tocilizumab treatment on serum myostatin in patients with RA.

Methods: 19 patients with RA (16 female, 3 male) mean age $49.6 y r s$, mean DAS28 6.1, median disease duration 10yrs (range 0 to 30 ), mean BMI 27.4, received $13 \mathrm{IV}$ infusions of TCZ $8 \mathrm{mg} / \mathrm{kg}$ every four weeks as part of a 52 week, single-centre, open-label study (ACT-NEUT [1]). 8 had previously been exposed to a biological agent, 4 were on regular steroids, and all patients received concurrent methotrexate. Serum myostatin was measured at 0,1,3 and 6months of treatment using ELISA. BMI, serum lipid profile, CRP, ESR and DAS28 were measured at each visit $(0,1,3$ and 6 months). Data were analysed using STATA 14. Change between 0,3 and 6 months was analysed using Wilcoxon signed rank test, statistical significance was confirmed using mixed model analysis adjusting for BMI, age and gender. Linear regression with adjustment for age, BMI and gender assessed correlation at baseline.

Results: DAS28, CRP and ESR all significantly decreased with TCZ treatment. A significant increase in BMI (25.5 (IQR 21.6-31.7) vs 26.0 (IQR 21.4-32.5) $\mathrm{p}=0.0052$ ) between baseline and 6 months and serum triglycerides between baseline and 3 months (1.3 (IQR 1.3-2.2) vs 1.6 (IQR 1.3-2.6) p=0.028) was seen with TCZ. Baseline serum myostatin concentrations were negatively correlated with baseline DAS28 $\left(r^{2}=0.39 \mathrm{p}=0.038\right)$. Treatment with tocilizumab for 6 months resulted in a significant increase in serum myostatin from baseline (2.7ng/mL (IQR 2.0-3.1) vs 3.3ng/mL (IQR 3.0-5.0) p <0.001) [Fig. 1]. Moreover, a significant association was seen between baseline myostatin and change in cholesterol at 3 months $\left(r^{2}=0.51 p=0.029\right)$, when adjusting for baseline cholesterol, age, gender, $\mathrm{BMI}$ and change in BMI.

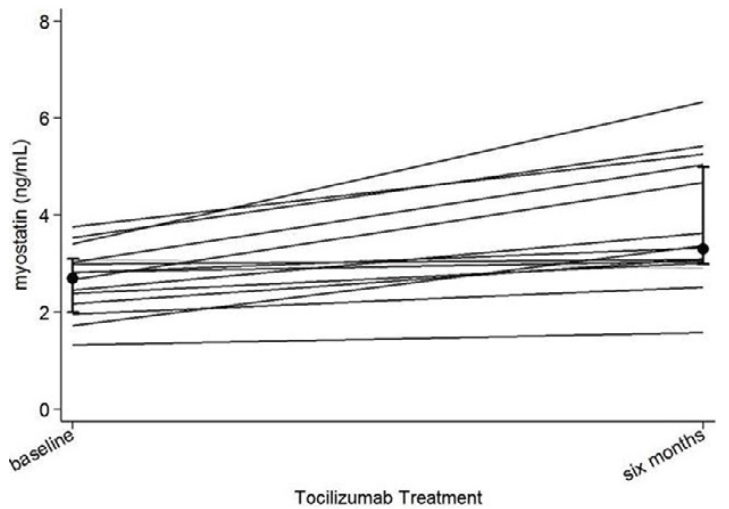

Fig 1. Serum myostatin levels significantly increase after six months of tocilizumab in patients with rheumatoid arthritis. Values shown are actual serum values for individual patients ( $\mathrm{n}=19$ at baseline $\mathrm{n}=15$ at 6 months). Black lines represent increasing serum values, grey lines decreasing values with treatment. Circles represent median $\pm I Q R$. Change evaluated by Wilcoxon signed rank test $\mathrm{p}<0.001$

Conclusions: We have demonstrated a significant correlation between myostatin and DAS28 and a significant change in myostatin with tocilizumab treatment. It is possible that IL- 6 blockade results in a rise in myostatin. This might attenuate any improvement in muscle wasting. Future studies should measure body composition and muscle function to help understand the changes we have observed.

\section{References:}

[1] Wright HL, Cross AL, Edwards SW, Moots RJ. Effects of IL-6 and IL-6 blockade on neutrophil function in vitro and in vivo. Rheumatology. 2014 Jul;53(7):1321-31.

Disclosure of Interest: None declared

DOI: 10.1136/annrheumdis-2017-eular.5831
SAT0210 IMPROVEMENT OF DISEASE ACTIVITY IN A 5-YEAR COHORT OF RHEUMATOID ARTHRITIS PATIENTS TREATED UNDER TREAT TO TARGET RECOMMENDATIONS RECEIVING BIOLOGICAL THERAPY

P. Santos-Moreno ${ }^{1}$, D. Gomez ${ }^{1}$, E. Castillo ${ }^{1}$, R. Giraldo ${ }^{1}$, G. Ballesteros ${ }^{1}$, L. Villarreal ${ }^{2}$, J. Bello ${ }^{3}$, D. Buitrago-Garcia ${ }^{3} .{ }^{1}$ Rheumatology; ${ }^{2}$ Psychology and processes; ${ }^{3}$ Epidemiology, Biomab, Center for Rheumatoid Arthritis, Bogota,

Bogota, Colombia

Background: Treat to Target (T2T) strategy becomes from the need to develop therapeutic targets and tools to achieve defined outcomes in rheumatoid arthritis (RA), this strategy has become recognized as a standard of good practice embodying the principle that rapid attainment of remission, or low disease activity, can halt joint damage and maintain good quality of life. However, there is no direct comparison between biologics in cohorts of patients with long-standing RA using T2T approach in real-life settings, which could have implications in treatment decisions and health economics.

Objectives: The aim of this study was to describe global change in Disease Activity Score 28 (DAS28) using T2T strategy for a 5 year period in patients with biological therapy in a large cohort of patients from a Colombian specialized in RA center with multidisciplinary approach

Methods: A descriptive dynamic cohort study was performed. Records of patients using biological DMARD treatment (Anti-TNF and others) from specialized in RA center were reviewed; those patients were followed-up under T2T standards. Clinical follow-up was according to DAS28 as follows: every $3-5$ weeks (DAS28 $>5.1$ ), every $7-9$ weeks (DAS28 $\geq 3.1$ and $\leq 5.1$ ), and every $11-13$ weeks (DAS28 $<3.1$ ). Therapy had to be adjusted with DAS28 $>3.2$ unless patient's conditions don't permit it. We divided patients in three groups: low disease activity (LDA), moderate disease activity (MDA) and severe disease activity (SDA) patients. Descriptive epidemiology was done, percentages and averages were calculated; the median of each variable was analyzed using t-Student assuming normality for DAS28 distribution and the level activity disease was analyzed using Pearson's statistics.

Results: During 60 month period we included 695 patients with biological therapy, $85 \%$ were female and $15 \%$ male. Mean age was 58 years \pm 11 . At beginning mean DAS28 was $4.1 \pm 1.1$ and $20 \%$ of patients were in SDA, and $55 \%$ in MDA. At the end of 5 year period mean DAS was $3.2 \pm 0.89$ and only $7 \%$ of patients were in SDA and $33 \%$ in MDA. The median was analyzed using t-Student assuming normality for DAS28 distribution. It showed statistical significant improvement $(p<0.00)$

\begin{tabular}{|l|r|r|r|r|}
\hline ACTIVITY LEVEL & TIME 0 n(\%) & $\begin{array}{l}2011-2012 \\
\mathrm{n}(\%)\end{array}$ & $2013-2014(\%)$ & 2015-2016(\%) \\
\hline REM & & $50(7)$ & $166(24)$ & $217(31)$ \\
\hline LDA & $178(26)$ & $236(34)$ & $181(26)$ & $198(28)$ \\
\hline MDA & $382(55)$ & $361(52)$ & $278(40)$ & $234(74)$ \\
\hline SDA & $135(19)$ & $98(14)$ & $70(10)$ & $46(7)$ \\
\hline
\end{tabular}

Conclusions: There was a global improvement of DAS28 in a cohort of RA patients receiving biological therapy followed and treated under recommendations of T2T approach demonstrating that with the strategies mentioned above it is possible to obtain a good control of the activity of the disease.

Disclosure of Interest: None declared

DOI: 10.1136/annrheumdis-2017-eular.5579

\section{SAT0211 REAL WORLD DATA OF RITUXIMAB EFFECTIVENESS IN RHEUMATOID ARTHRITIS: DIFFERENCES BETWEEN BIOLOGIC-NAIVE PATIENTS AND PREVIOUSLY EXPOSED TO BIOLOGICS}

R.M. Ferreira, R. Fonseca, D.R. Gonçalves, F. Aguiar, T.M. Rocha, A. Bernardo, S. Pimenta, M. Bernardes, L. Costa. São João Hospital Centre, Porto, Portugal

Background: Rituximab is only approved for rheumatoid arthritis (RA) treatment in patients with an incomplete response or intolerance to others DMARDs, including TNF alfa inhibitors. It represents a significant advance in RA biologics arsenal due to its safety and efficacy profiles.

Objectives: To evaluate the effectiveness of rituximab in RA patients and to compare the response between first-line rituximab patients and those previously exposed to other biologics.

Methods: An observational retrospective study was conducted, including all the consecutive patients with diagnosis of RA under rituximab, followed at our Rheumatology department until December 2016. Demographic and clinical data were obtained by consulting the national database (Reuma.pt). DAS28 variations and EULAR response were measured at 6,12 and 18 months. Parametric and non parametric tests were used for statistics (SPSS 20.0).

Results: We included 63 RA patients ( $81 \%$ of women), with a mean (SD) age of 61 (10) years and a mean disease duration of $19(10)$ years; $86 \%$ rheumatoid factor positive and $87 \%$ anti- citrullinated peptide antibody (ACPA) positive. Bone erosions and extra-articular manifestations were present in $85,7 \%$ and $58,7 \%$ of the patients, respectively. At baseline, the mean DAS28 was $5.79(65 \%$ and $29 \%$ of patients with severe and moderate disease activity, respectively, and $6 \%$ in clinical remission). Thirty patients were treated with rituximab as first-line therapy and 33 patients were previously exposed to other biologics. Combination therapy 